\title{
EXTENSIÓN DE LA FRONTERA MARÍTIMA ENTRE PERÚ Y CHILE. LA EQUIDAD COMO CONCEPTO DECISORIO. Disputa marítima, Perú $V$. Chile CORTE INTERNACIONAL DE JUSTICIA
}

\section{DANiEl PARodi Nebreda*}

\section{INTRODUCCIÓN}

Con fecha 27 de enero de 2014, la Corte Internacional de Justicia (en adelante indistintamente la "CIJ" o la "Corte") ha dado a conocer su fallo respecto de la disputa marítima que la República de Perú (en adelante "Perú") interpuso en contra de la República de Chile (en adelante "Chile" y conjuntamente con Perú las "Partes") respecto de la delimitación de la frontera marítima entre ambos Estados.

En breve, Perú solicitó a la Corte (i) la determinación del curso de la frontera marítima entre ambos Estados de acuerdo al derecho internacional, teniendo como punto de partida el "Punto Concordia" y equidistante desde las líneas de bases de ambas Partes hasta un punto situado a una distancia de 200 millas náuticas de aquellas líneas de bases; y (ii) la adjudicación y declaración de que Perú posee derechos soberanos exclusivos sobre el área marítima situada al interior de límite de 200 millas náuticas desde su costa pero fuera de la zona económica exclusiva de Chile.

Chile responde solicitando que se (i) desestime el total de las solicitudes de Perú, y (ii) adjudique y declare que: (a) los títulos a la respectiva zona marítima de Chile y Perú han sido totalmente delimitados por acuerdo; (b) esas zonas marítimas se encuentran delimitadas por una frontera que sigue un paralelo de latitud pasando desde el punto conoci-

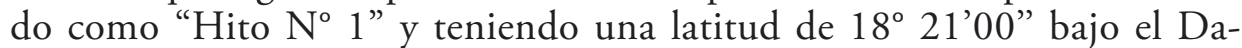
tum WGS 84; y (c) Perú no tiene título alguno a la zona marítima que se extiende bajo ese paralelo.

El elemento fundamental que la Corte tuvo que resolver fue la existencia o ausencia de un tratado que estableciera la frontera marítima compartida, situación que la Corte resolvió positivamente, pero dándole caracteres limitados en cuanto a su proyección geográfica.

El párrafo primero del artículo 38 del Estatuto de la Corte Internacional de Justicia, dispone que la función de la Corte es decidir conforme al derecho internacional las controversias que le sean sometidas, estable-

Abogado. Licenciado en Derecho, Pontificia Universidad Católica de Chile (CHIle). Abogado asociado de Philippi Irarrázaval Pulido \& Brunner Abogados. Correo electrónico: diparodi@uc.cl. 
ciendo que para ello esta deberá aplicar: $a$. las convenciones internacionales, sean generales o particulares, que establecen reglas expresamente reconocidas por los Estados litigantes; $b$. la costumbre internacional como prueba de una práctica generalmente aceptada como derecho; $c$. los principios generales de derecho reconocidos por las naciones civilizadas; $y d$. las decisiones judiciales y las doctrinas de los publicistas de mayor competencia de las distintas naciones, como medio auxiliar para la determinación de las reglas de derecho.

A su vez el párrafo segundo del citado Estatuto dispone que ello no restringe la facultad de la Corte para decidir un litigio ex aequo et bono, si las partes así lo convinieren. Bien nos parece señalar que, siguiendo a Yrarrázaval, entendemos el concepto de "ex aequo et bono" como, la equidad y el leal saber y entender ${ }^{1}$.

Perú y Chile no convinieron que la Corte resolviera ex aquo et bono, y por lo mismo esta se encontraba en la obligación de encontrar la solución al juicio en los elementos descritos por el párrafo primero del artículo 38 de su estatuto. No podía la Corte buscar la solución en la equidad. Sin perjuicio de lo anterior, parte de la doctrina considera que la equidad se encuentra dentro del concepto de "principios generales del derecho" 2 .

Como se detallará más adelante la Corte dispuso la existencia de una frontera marítima entre las Partes, concluyendo que esta se divide en tres secciones: la primera, establecida por un acuerdo tácito entre las partes, que comienza en la intersección del paralelo que pasa por el Hito $\mathrm{N}^{\circ} 1$ con el punto de bajamar y se extiende por 80 millas náuticas sobre dicho paralelo hasta el Punto A. Desde el Punto A la frontera sigue una línea equidistante en dirección general suroeste hasta el Punto B. Finalmente la frontera continúa en dirección general sur desde el Punto B a lo largo del límite de 200 millas náuticas desde la costa chilena hasta el Punto C.

Dividiremos en tres las cuestiones a tratar en el presente artículo, primero la existencia de un límite marítimo entre Chile y Perú, segundo, la extensión de dicho límite previamente establecido por las Partes y finalmente la delimitación de aquella sección de la frontera que, a criterio de la Corte, no se encontraba regulada por acuerdo de las Partes. Entregaremos una visión crítica del procedimiento utilizado por y del resultado final al que llega la Corte en este caso en particular a la luz del texto del fallo, de las opiniones minoritarias de jueces que no compartieron todos los elementos dispositivos de la sentencia, del derecho internacional y de

Yrarrázaval C., Arturo (2012): Diccionario Jurídico-Económico (Santiago, Ediciones Universidad Católica de Chile, $1^{\text {a }}$ edición), p. 348.

White, Margaret (2004): "Equity: A General Principle of Law Recorgnised by Civilised Nations?”, Law and Justice Journal, Vol. $4 \mathrm{~N}^{\circ}$ 1, 2005: p. 116. 
otros fallos de la Corte sobre materias similares, con el fin de explicar y criticar el resultado final de la disputa ${ }^{3}$.

Finalmente analizaremos y criticaremos que, al igual que en otros casos, la Corte enmarcó dentro de los principios generales del derecho internacional los "principios equitativos", es decir conceptualizó la equidad como principio y de esa forma se autorizó a sí misma para fallar de una forma que parece ser más cercana al numeral segundo que al primero del artículo 38 de su Estatuto.

\section{1) Existencia de Límite Marítimo entre Chile y Perú}

En la siguiente sección analizaremos uno por uno parte de los elementos de hecho que la Corte revisó para llegar a sus conclusiones.

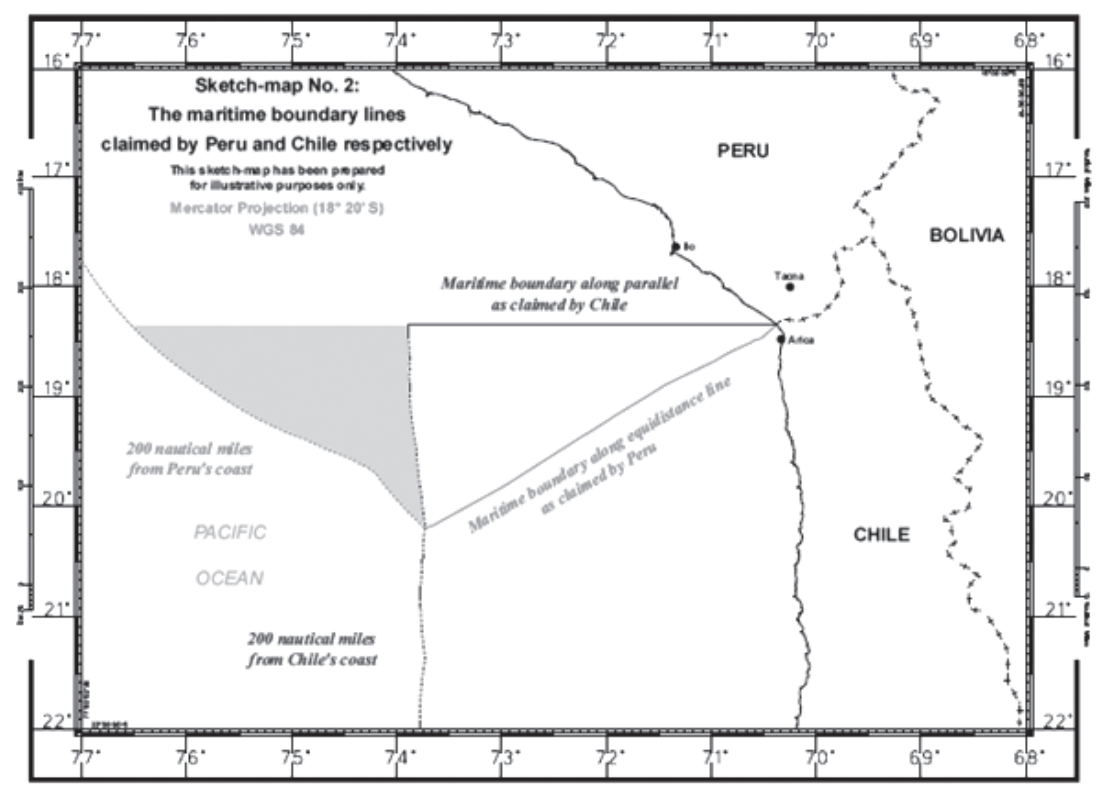

\section{1) Proclamaciones de 1947 de Chile y Perú}

Con fecha 23 de junio y 1 de agosto de 1947, Chile y Perú emitieron respectivamente proclamaciones oficiales, cada Estado reclamando una

3 Nota del Autor. (1) Los textos de la sentencia y de las opiniones minoritarias de los jueces se encuentran solo disponibles en idioma inglés, en razón de lo anterior las citas a aquellos documentos que se incorporan al presente trabajo son traducciones libres realizadas por el autor. (2) Los mapas contenidos en el presente trabajo son aquellos preparados por la Corte para la sentencia en cuestión. 
zona marítima de 200 millas marinas desde su costa. Las Partes reclamaron "la soberanía nacional" en el caso de Chile, y "la soberanía y la jurisdicción nacionales" en el caso del Perú, sobre la plataforma continental y las aguas adyacentes a sus respectivas costas, como asimismo el control sobre todos los recursos naturales contenidos en ellas. Entre una y otra proclamación mediaron menos de seis semanas. Chile tomó primero la iniciativa. Ambas fueron formuladas en términos sustancialmente similares ${ }^{4}$.

Las proclamaciones fueron emitidas en respuesta a la pesca y caza industrial de ballenas por flotas extranjeras. Las Partes consideraron injusta esa explotación de sus recursos naturales ya que los Estados ribereños no obtenían beneficio alguno y sus propias industrias no estaban suficientemente desarrolladas como para hacer posible la pesca y caza de ballenas a semejante escala ${ }^{5}$.

La Corte nota que las mencionadas proclamaciones no establecen en sí mismas una frontera marítima internacional si no que se preocupan más de establecer la prolongación del área marítima sujeta al control de cada Estado, sin otorgarle importancia a los límites laterales de esta y, en virtud de ello, la Corte no les otorga el valor de crear una frontera común ${ }^{6}$.

Efectivamente las proclamaciones de ambos países parecen ser más que nada un paso inicial e innovador hacia la creación de un nuevo tipo de zona marítima bajo la jurisdicción del Estado ribereño que un instrumento de demarcación territorial. Cabe hacer presente que la doctrina internacional le da a esta proclamación una gravitante importancia en el desarrollo del derecho del mar moderno y en particular en la creación de lo que hoy conocemos como Zona Económica Exclusiva7 89.

\section{2) Declaración de SANTIAgo de 1952}

En 1952 Chile, Perú y Ecuador sostuvieron la Conferencia para el Explotación y Conservación de los Riquezas Marítimas del Pacífico Sur, en la cual suscribieron cuatro instrumentos, entre los cuales se encontraba la Declaración de Santiago.

Contramemoria de Chile para el Caso de Delimitación Marítima (Perú v. Chile), p. 43. Contramemoria de Chile para el Caso de Delimitación Marítima (Perú v. Chile), p. 44. International Court of Justice, Maritime Dispute (Perú v. Chile), January 2014, Judgment; $\$ 39$.

7 Benadava, Santiago (2005): Derecho Internacional Público (Santiago, Editorial LexisNexis, octava edición), p. 258.

8 Orrego, Francisco (1991): La Zona Económica Exclusiva: Régimen y Naturaleza Jurídica en el Derecho Internacional (Santiago, Editorial Jurídica de Chile, $1^{a}$ edición), p. 11.

9 Llanos, Hugo (2007): Teoría y Práctica del Derecho Internacional Público, El Estado como sujeto de Derecho Internacional, Tomo II, Volumen I (Santiago, Editorial Jurídica de Chile, $3^{\text {a }}$ edición), p. 317. 
En la Declaración de Santiago los Gobiernos de Chile, Perú y Ecuador proclamaron como norma de su política internacional marítima, la soberanía y jurisdicción exclusivas que a cada uno de ellos corresponde sobre el mar que baña las costas de sus respectivos países, hasta una distancia mínima de 200 millas náuticas desde las referidas $\operatorname{costas}^{10}$.

La Declaración de Santiago no hace referencia expresa a la delimitación de fronteras marítimas entre las Partes y, a pesar de ser un tratado que hace referencia a la proyección marítima (200 millas náuticas desde la costa), no es elemento suficiente para determinar la existencia de una frontera marítima por acuerdo entre las Partes ${ }^{11}$.

Esta declaración es probablemente el acto plurinacional más importante en el desarrollo del concepto jurídico de Zona Económica Exclusiva, es este el primer impulso conjunto de Estados en vías de desarrollo para proteger sus recursos marítimos de las flotas pesqueras de largo alcance de países desarrollados, equipadas con procesadores de pescado, radares y frigoríficos que realizaban faenas de extracción en zonas de alta mar cercanas a sus territorios ${ }^{12}$.

\section{3) Convenio sobre Zona Especial Fronteriza Marítima de 1954}

Entre los varios acuerdos celebrados por Chile, Perú y Ecuador en 1954, la argumentación chilena hace especial énfasis en el Convenio sobre Zona Especial Fronteriza Marítima de fecha 4 de diciembre de 1954, en adelante el "Convenio".

Señala la defensa chilena que este acuerdo reconoce implícitamente que a la fecha de su celebración existían fronteras marítimas entre las Partes. Así se cita el considerando del Convenio al establecer "Que la experiencia ha demostrado que debido a las dificultades que encuentran las embarcaciones de poco porte tripuladas por gente de mar con escasos conocimientos de náutica o que carecen de los instrumentos necesarios para determinar con exactitud su posición en alta mar, se producen con frecuencia, de modo inocente y accidental, violaciones de la frontera marítima entre los Estados vecinos; Que la aplicación de sanciones en estos casos produce siempre resentimientos entre los pescadores y fricciones entre los países que pueden afectar al espíritu de colaboración y de unidad que en todo momento debe animar a los países signatarios de los acuerdos de Santiago; y Que es conveniente evitar la posibilidad de estas involuntarias

\footnotetext{
10 Declaración de Santiago celebrada entre Chile, Perú y Ecuador (1952), sección II.

11 International Court of Justice, Maritime Dispute (Perú v. Chile), January 2014, Judgment; $\$ 27$.

12 Benadava (2005), p. 267.
} 
infracciones cuyas consecuencias sufren principalmente los pescadores" 13 . (Énfasis agregado).

El citado Convenio dispone en sus artículos primero y segundo el establecimiento de una zona especial, a partir de las 12 millas marinas de la costa, de 10 millas marinas de ancho a cada lado del paralelo que constituye el límite marítimo entre los dos países; y que la presencia accidental en la referida zona de las embarcaciones de cualquiera de los países limítrofes aludida en el primer considerando, no será considerada como violación de las aguas de la zona marítima, sin que esto signifique reconocimientos de derecho alguno para ejercer faenas de pesca o caza con propósito preconcebido en dicha Zona Especial.

La Corte expresa que el Convenio no indica cuándo y mediante qué medios se acordó la ya establecida frontera marítima. La Corte señala que el reconocimiento por las Partes de esta frontera solo puede demostrar que Chile y Perú habían previamente llegado a un acuerdo tácito ("tacit agreement") respecto de la frontera marítima que comparten ${ }^{14}$.

\section{4) LOS ACUERDOS DE 1968 Y 1969 RESPECTO DE FAROS DE ENFILACIÓN}

En 1968 y 1969 Perú y Chile acordaron establecer "marcas de enfilación visibles desde el mar, que materialicen el paralelo de la frontera marítima que se origina en el Hito número uno ( $\left.\mathrm{N}^{\circ} .1\right)$ " con el fin de permitir a las naves que navegaren a pocos kilómetros de la costa distinguir la frontera entre ambos países.

Al igual que en el caso del Convenio estos acuerdos demuestran, conforme al criterio de la Corte, la existencia a la fecha de su suscripción de una frontera marítima entre Perú y Chile, y además agregan que dicha frontera se origina en el punto en que el paralelo que pasa por el Hito $\mathrm{N}^{\circ} 1$ toca la línea de bajamar ${ }^{15}$.

\section{5) Conclusiones de la CORTE}

La Corte concluye que, a pesar de no existir un tratado limítrofe propiamente tal entre las Partes del litigio, ya en 1954 existía entre Chile y Perú un acuerdo tácito que establecía la frontera marítima entre ambos países, consistente en el paralelo que se origina en el punto en que el pa-

\footnotetext{
13 Convenio sobre Zona Especial Fronteriza Marítima celebrado entre Chile, Perú y Ecuador (1954), sección de considerandos.

14 International Court of Justice, Maritime Dispute (Perú v. Chile), January 2014, Judgment; $\$ 91$.

15 International Court of Justice, Maritime Dispute (Perú v. Chile), January 2014, Judgment; $\$ 174$.
} 
ralelo que pasa por el Hito $\mathrm{N}^{\circ} 1$ llega a la línea de bajamar. La Corte llega al acuerdo de que esta es una frontera para todo propósito que rige para el suelo marino y el subsuelo al igual que para las aguas sobre ellos puesto que a pesar que el Convenio y los acuerdos de 1968 y 1969 tienen fines particulares no buscan crear una delimitación territorial para un asunto en específico, sino que tienen la intención de generar regulaciones para asuntos particulares usando como referencia la delimitación fronteriza ya existente entre las Partes ${ }^{16}$.

\section{2) Extensión de la Frontera Marítima acordada por Perú y CHILE}

La Corte determina que la frontera marítima acordada tácitamente entre Chile y Perú se extiende exclusivamente por 80 millas náuticas y que a mayor distancia de ese punto las Partes del litigio no han acordado frontera alguna. Los argumentos esgrimidos por la CIJ son, entre otros, los siguientes:

2.1) El Convenio y los acuerdos de 1968 y 1969 se refieren a la regulación de la pesca y la navegación de naves locales a pocas millas de tierra firme. La evidencia presentada por las Partes respecto de las actividades pesqueras, a la época de los mencionados instrumentos, indica que era improbable que estas se desarrollaran en toda la extensión de las $200 \mathrm{mi}-$ llas náuticas y, por lo mismo, que las Partes hayan tenido la intención de regular la mencionada frontera en toda esa extensión ${ }^{17}$.

2.2) Sobre la base de las actividades pesqueras de las Partes en dicha época, la práctica relevante de otros Estados y el trabajo de la Comisión de Derecho Internacional sobre Derecho del Mar, la Corte considera que la frontera marítima acordada entre Chile y Perú no podría haberse extendido más allá de 80 millas náuticas desde su punto de partida ${ }^{18}$.

2.3) La mayor parte de las actividades de control realizadas por las Partes en la zona marítima fronteriza hasta mediados de la década de

\footnotetext{
16 International Court of Justice, Maritime Dispute (Perú v. Chile), January 2014, Judgment; $\$ 100-102$.

17 International Court of Justice, Maritime Dispute (Perú v. Chile), January 2014, Judgment; $\$ 111$.

18 International Court of Justice, Maritime Dispute (Perú v. Chile), January 2014, Judgment; $\$ 117$.
} 
1980 estaba dentro de un radio de 60 millas náuticas desde tierra firme y usualmente mucho más cerca ${ }^{19}$.

2.4) No fue sino hasta 1982 con la celebración de la Convención de las Naciones Unidas sobre el Derecho del Mar que se reconoció legalmente la existencia de una zona económica exclusiva existente hasta la milla 200 desde tierra firme, mal podrían las Partes haber establecido una frontera que a aquella época no era reconocida por el Derecho Internacio$\mathrm{nal}^{20}$.

La Corte concluye que lo anterior dejaba cierta incertidumbre ("some uncertainty") respecto de la extensión precisa de la frontera marítima acordada. Sin embargo, la Corte concluye que la frontera marítima acordada entre las Partes se extiende por una distancia de 80 millas náuticas desde el punto en que el paralelo que pasa por el Hito $\mathrm{N}^{\circ} 1$ llega a bajamar ${ }^{21}$.

Es precisamente en esta sección de la sentencia de la Corte que encontramos elementos a comentar y criticar.

Es la misma Corte la que señala la existencia de cierta incertidumbre respecto de la extensión de la frontera acordada por las Partes. La elección de 80 millas parece, a todas luces, arbitraria, puesto que dentro de la evidencia analizada por la Corte y de las argumentaciones planteadas por esta, no aparece elemento alguno que demuestre que las Partes acordaron dicho número de millas como el término de su frontera común. La Corte podría haberle otorgado una extensión de 100, 50 millas náuticas o bien cualquier número o fracción de número de millas náuticas entre ellas. Al respecto algunos puntos a destacar:

i) Ninguno de los tratados, acuerdos y declaraciones analizados por la Corte contiene referencias a la milla 80 , ni siquiera hay menciones al área comprendida entre el fin del mar territorial (12 millas náuticas) y las 200 millas náuticas. No hay elemento de texto alguno que permita concluir que las Partes acordaron la ya mencionada extensión. Como razona el juez Skotnikov en su opinión parcialmente disidente, la evidencia no demuestra que la extensión de la frontera marítima debe haber sido 80 millas náuticas, en oposición a cualquier otro número ${ }^{22}$. Ninguna de las Partes en sus presentaciones declara que la milla 80 sea el límite acordado

19 International Court of Justice, Maritime Dispute (Perú v. Chile), January 2014, Judgment; $\$ 128$.

20 International Court of Justice, Maritime Dispute (Perú v. Chile), January 2014, Judgment; $\$ 115$.

21 International Court of Justice, Maritime Dispute (Perú v. Chile), January 2014, Judgment; $\$ 151$.

22 Sкотnikov, Leonid; Partly Dissenting Opinion regarding the Maritime Dispute (Perú v. Chile), January 2014, Judgment; $\$ 6$. 
de la frontera que las une y menos hace petitorios a la Corte que la declare como tal.

La jueza Sebutinde es más drástica en su opinión minoritaria al razonar que no le es claro cómo la conclusión de la Corte de que, dada la práctica de las Partes del litigio, estas no pudieron haber acordado tácitamente una frontera marítima con una extensión mayor de 80 millas náuticas pueda convertirse en un hallazgo legal de que las Partes acordaron una frontera de 80 millas náuticas. Sebutinde agrega que, en su opinión, este hallazgo de la Corte descansa sobre terrenos peligrosamente débiles y especulativos ${ }^{23}$.

ii) La práctica pesquera de las Partes en la década de 1950 era de pequeńa escala y limitada principalmente a un área que no se extendía más allá de las 60 millas náuticas de la costa debido a las limitadas capacidades industriales de ambos Estados.

No puede la Corte desprender que las limitaciones industriales y económicas de un Estado le impidan regular sus fronteras más allá de las áreas en que le es posible realizar actividades extractivas. La delimitación de fronteras es una actividad que deriva del principio de soberanía territorial de los Estados y no puede verse limitada por las capacidades económicas e industriales de estos. Como dice el Presidente Tomka en su opinión parcialmente disidente "las fronteras no se establecen solo para pescadores que conducen sus actividades desde botes pequeños. Las fronteras sirven propósitos más generales" 24 .

En este aspecto la Corte ha tenido una oscilante opinión puesto que parte de su jurisprudencia le resta importancia a la conducta de los Estados litigantes para efectos de delimitación fronteriza a menos que dicha conducta tenga un carácter excepcional ${ }^{25}$.

A mayor abundamiento, la doctrina internacional es prácticamente unánime en que las declaraciones y convenciones analizadas por la Corte tenían la intención de proteger un área de 200 millas náuticas específicamente porque los Estados en cuestión no tenían la capacidad industrial para explotarla por sí mismos. Los instrumentos en cuestión fueron creados para proteger un área que las Partes consideraban suya pero que era explotada por terceros Estados más desarrollados, por lo mismo la inten-

\footnotetext{
23 Sebutinde, Julia; Partly Dissenting Opinion regarding the Maritime Dispute (Perú v. Chile), January 2014, Judgment; $\$ 13$.

24 Tomka, Peter; Partly Dissenting Opinion regarding the Maritime Dispute (Perú v. Chile), January 2014, Judgment; $\$ 2$.

25 Reichler, Paul (2013): "A Case of Equitable Maritime Delimitation: Nicaragua and Colombia in the Western Caribbean Sea", Revista Tribuna Internacional, vol. 2, № 3, p. 145.
} 
ción de las Partes era exactamente opuesta a la conclusión a la que llegó la Corte.

La Corte incurre en contradicciones en su argumentación:

i) A la fecha de los instrumentos el Derecho Internacional no reconocía las 80 millas náuticas como una extensión válida de la frontera marítima entre dos Estados. Si las Partes del litigio, según argumenta la Corte, no podían establecer las 200 millas como la extensión de su frontera en razón de que el Derecho Internacional no lo reconocía, tampoco podrían haberla fijado en 80 millas.

Al respecto el juez $A d$ hoc Orrego sostiene en su opinión parcialmente disidente que "no comparte el entendimiento limitado de la Corte puesto que los instrumentos eran en cualquier caso capaces de acordar respecto una delimitación marítima de tres Estados respecto de su derechos potenciales"; añade Orrego que los citados instrumentos "son aquellos actos gatillantes de un desarrollo que, después de una evolución sistemática, llevaron al concepto de zona económica exclusiva y otros conceptos claves del derecho del mar vigente hoy recogido por la Convención de Naciones Unidas sobre el Derecho del Mar de 1982 y reconocida por esta Corte como parte del derecho internacional consuetudinario" ${ }^{26}$.

El juez Skotnikov es de la opinión que las Proclamaciones de 1947 y la Declaración de Santiago de 1952 demuestran que las Partes tenían la intención de hacer reclamos marítimos que no gozaban de aceptación contemporánea internacionalmente generalizada. Sin perjuicio de lo anterior, el establecimiento de una frontera marítima en la década de 1950 solo puede ser entendido como un acuerdo inter partes, ejecutable primariamente inter se. Lo anterior no puede tener el carácter controversial que le otorga la Corte ${ }^{27}$.

ii) La Corte concluye que la frontera marítima acordada por acuerdo tácito entre Chile y Perú es una frontera para todo propósito (all-purpose boundary). Sin perjuicio de lo anterior, la Corte señala que la práctica pesquera de los Estados le permite llegar a la conclusión que las Partes no podrían haber establecido una frontera común pasadas las 80 millas náuticas. El argumento de la Corte nos parece contradictorio puesto que es difícil de entender que una frontera para todo propósito pueda ser limitada por una actividad extractiva en particular.

Similar es la opinión de Skotnikov quien señala que no logra entender cómo es que una frontera para todo propósito pueda ser determinada por la capacidad extractiva y de control de las Partes al tiempo de la firma

\footnotetext{
26 Orrego, Francisco; Partly Dissenting Opinion regarding the Maritime Dispute (Perú v. Chile), January 2014, Judgment; $\$ 23$.

27 Sкотnikov, Leonid; Partly Dissenting Opinion regarding the Maritime Dispute (Perú v. Chile), January 2014, Judgment; $\$ 4$.
} 
del Convenio de 1954, siendo que dicho convenio meramente reconoce la frontera marítima ya existente ${ }^{28}$.

\section{3) Delimitación de la Frontera Marítima entre Chile y Perú}

La Corte delimitó la frontera comprendida entre 80 y 200 millas náuticas desde la costa de conformidad a las reglas que establece la Convención de Naciones Unidas sobre el Derecho del Mar de 1982, en adelante "UNCLOS", para la delimitación fronteriza en la zona económica exclusiva. La norma contenida en el párrafo primero del artículo 74 de UNCLOS es la destinada a resolver el asunto en cuestión, toda vez que dispone "La delimitación de la zona económica exclusiva entre Estados con costas adyacentes o situadas frente a frente se efectuará por acuerdo entre ellos sobre la base del derecho internacional, a que se hace referencia en el artículo 38 del Estatuto de la Corte Internacional de Justicia, a fin de llegar a una solución equitativa." (Énfasis agregado).

La metodología que la Corte usualmente ${ }^{29}$ ocupa para llegar a una solución equitativa se compone de tres etapas: Primero construye una línea equidistante provisional, a menos que hayan argumentos sólidos que sugieran lo contrario; segundo, considera si es que hay circunstancias relevantes que puedan sugerir ajustes a la línea para obtener una solución equitativa; y finalmente la Corte conduce un test de desproporcionalidad en el cual evalúa si es que el efecto de la línea es tal que las respectivas áreas otorgadas a las Partes sean marcadamente desproporcionadas a los largos de sus costas relevantes ${ }^{30}$.

$\mathrm{Al}$ respecto de la metodología utilizada por la Corte, Perú ha señalado tres puntos. Primero, debe construirse una línea equidistante entre las costas de ambos países; segundo, no hay circunstancias especiales que lleven a ajustar la línea equidistante; y tercero, la aplicación del test de desproporcionalidad ex post confirma la naturaleza equitativa de la líneas equidistante. Chile no incorporó argumentos respecto de esta materia puesto que su posición fue que las Partes ya habían delimitado la frontera

28 Sкотnikov, Leonid; Partly Dissenting Opinion regarding the Maritime Dispute (Perú v. Chile), January 2014, Judgment; $\$ 5$.

29 La Corte ha usado este método en múltiples ocasiones, siendo el antecedente más relevante para este caso la disputa territorial y marítima entre Nicaragua y Colombia fallada en 2013. La misma Corte reconoce en su fallo de 2009 para la Delimitación Marítima del Mar Negro que este método es parte de su jurisprudencia asentada (“Settled jurisprudence") (International Court of Justice, Maritime Delimitation of the Black Sea (Rumania v. Ukraine), February 2009, Judgment; $\$ 118$.

30 International Court of Justice, Maritime Dispute (Perú v. Chile), January 2014, Judgment; $\$ 180$. 
marítima en cuestión y que por lo tanto la Corte no tendría que realizar proceso de delimitación alguno ${ }^{31}$.

La Corte selecciona el punto que considera como el fin de la frontera acordada por las Partes, el "Punto A", como el lugar de origen de la línea de equidistancia. La Corte dispone un número de puntos base en las respectivas costas de ambos países, el primer punto base peruano se encuentra a 80 millas náuticas del Punto A con el fin de situarlo a una distancia idéntica de la que separa el Punto A del punto de partida de la frontera acordada tácitamente entre las Partes. A partir del Punto A, la Corte dibuja una línea equidistante desde las costas de ambos Estados que continúa en dirección general suroeste hasta el punto en que se encuentra con el límite de 200 millas náuticas desde la costa de Chile, el "Punto B".

La Corte opina que en el presente caso la línea equidistante evita cualquier amputación excesiva de las proyecciones marítimas de cualquiera de las Partes y que no se han presentado circunstancias relevantes que aconsejen alterar la línea equidistante. A su vez concluye que no existe una desproporción significativa entre las áreas marítimas asignadas a cada Parte en virtud de la línea equidistante ${ }^{32}$.

En adición, la Corte dispone que no es necesario fallar respecto de la solicitud de Perú de asignarle toda el área marítima que se encuentre dentro de las 200 millas náuticas desde su costa pero que se encuentra fuera de las 200 millas náuticas desde la costa chilena, el llamado triángulo exterior o triángulo de altamar. Esto en virtud de que dicha área reclamada por Perú queda, a criterio de la Corte, fuera de las áreas de interés superpuestas. Lo anterior genera el punto final en que Chile y Perú comparten frontera marítima, el "Punto C"33.

De esta forma la Corte concluye que la frontera marítima entre las Partes se divide en tres secciones, la primera que comienza en la intersección del paralelo que pasa por el Hito $\mathrm{N}^{\circ} 1$ con el punto de bajamar y se extiende por 80 millas náuticas sobre dicho paralelo hasta el Punto A. Desde el Punto A la frontera sigue una línea equidistante de las costas de ambos Estados en dirección general suroeste hasta el Punto B. Finalmente la frontera continúa en dirección general sur desde el Punto $\mathrm{B}$ a lo largo del límite de 200 millas náuticas desde la costa chilena hasta el Punto C.

\footnotetext{
31 International Court of Justice, Maritime Dispute (Perú v. Chile), January 2014, Judgment; \$181-182.

32 International Court of Justice, Maritime Dispute (Perú v. Chile), January 2014, Judgment; $\$ 194$.

33 International Court of Justice, Maritime Dispute (Perú v. Chile), January 2014, Judgment; $\$ 188-190$.
} 


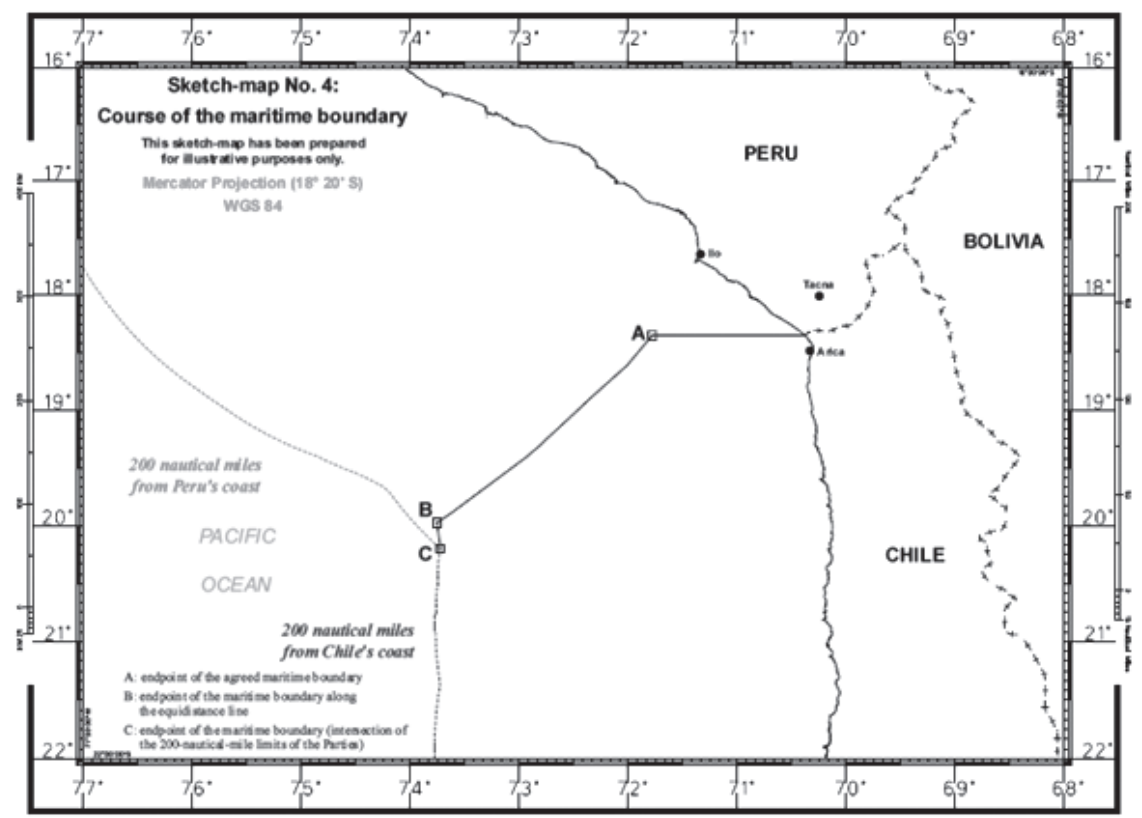

La metodología usada por la Corte para la delimitación de la frontera marítima entre Chile y Perú es efectivamente la que la Corte ha usado en casos anteriores, y en cuanto a su forma no nos merece comentarios pues parece ser, en principio, una forma correcta y justa de delimitación. Lo anterior, sin perjuicio de que, siguiendo al Presidente Tomka, al no estar de acuerdo en que la frontera acordada tácitamente entre las Partes se encuentre delimitada solo hasta 80 millas náutica desde su punto de partida, no podemos apoyar la subsecuente delimitación que hace la Corte respecto de la continuación de la frontera. No se critica la metodología de delimitación, sino que se critica el punto en que la línea equidistante se separa del paralelo ${ }^{34}$.

El juez Orrego critica la delimitación que hace la Corte entre el Punto A y el Punto C en razón de que la Corte no consideró el triángulo de alta mar en su test de desproporcionalidad, asignándole una gran cantidad de kilómetros cuadrados a Perú, de conformidad con su pretensión, y restándole una considerable cantidad a Chile, negando parcialmente sus pretensiones.

34 Tomka, Peter; Partly Dissenting Opinion regarding the Maritime Dispute (Perú v. Chile), January 2014, Judgment; $\$ 25$. 


\section{4) La EQUidad COMO FACTOR DeCISORIO}

Es importante para estos efectos considerar una reflexión que hizo la Corte en el fallo de 24 de febrero de 1982 respecto de la disputa sobre la Plataforma Continental entre Túnez y la Jamahiriya Árabe Libia en la cual dispone que "(...) el resultado de la aplicación de principios equitativos debe ser equitativa. Esta terminología, que es generalmente usada, no es completamente satisfactoria puesto que emplea el término "equitativo" para describir tanto el resultado a ser logrado como los medios que deben ser aplicados para obtener dicho resultado. Es, sin embargo, el resultado el que debe predominar; los principios se encuentran subordinados a la meta. La equitatividad de un principio debe ser evaluada a la luz de su utilidad para poder llegar a un resultado equitativo. No todo principio es en sí mismo equitativo, pero puede adquirir dicha cualidad por referencia a la equitatividad de la solución. Los principios a ser señalados por la Corte deben ser seleccionados de acuerdo a su capacidad para obtener un resultado equitativo" 35 .

En el mismo fallo de 1982 la Corte define que "la equidad como concepto legal es una emanación directa de la idea de Justicia. La Corte se encuentra obligada a aplicar la equidad equitativa como parte del derecho internacional general ${ }^{36}$. Según la Corte, su jurisprudencia muestra que en disputas respecto de delimitación marítima la equidad no es un método de delimitación, pero simplemente una meta que debiese ser tenida presente al efectuar la delimitación ${ }^{37}$. Similar reflexión hace la Corte en 1969 en el fallo respecto del caso sobre la Plataforma Continental del

35 International Court of Justice, Continental Platform Dispute (Tunisia v. Libyan Arab Jamahiriya), February 1982, Judgment; $\$ 70$ "The result of the application of equitable principles must be equitable. This terminology, which is generally used, is not entirely satisfactory because it employs the term equitable to characterize both the result to be achieved and the means to be applied to reach this result. It is, however, the result which is predominant; the principles are subordinate to the goal. The equitableness of a principle must be assessed in the light of its sefulness for the purpose of arriving at an equitable result. It is not every such principle which is in itself equitable; it may acquire this quality by reference to the equitableness of the solution. The principles to be indicated by the Court have to be selected according to their appropriateness for reaching an equitable result".

36 International Court of Justice, Continental Platform Dispute (Tunisia v. Libyan Arab Jamahiriya), February 1982, Judgment; $\$ 71$ "Equity as a legal concept is a direct emanation of the idea of Justice. The Court is bound to apply equitable equity as a part of general international law".

37 International Court of Justice, Land and Maritime Boundary Case (Camerún v. Nigeria), Octubre 2002, Judgment; $\$ 294$ "The Court is bound to stress in this connection that delimiting with a concern to achieving an equitable result, as required by current international law, is not the same as delimiting in equity. The Court's jurisprudence shows that, in disputes relating to maritime delimitation, equity is not a method of delimitation, but solely an aim that should be borne in mind in effecting the delimitation". 
Mar del Norte. En efecto la Corte dispone que es necesario buscar no un método de delimitación, sino que una meta ${ }^{38}$.

Las reflexiones que hace la Corte en los fallos citados más arriba son de gran importancia para efectos de entender el resultado del litigio entre Perú y Chile. De acuerdo a lo considerado y fallado por la Corte podemos desprender dos conclusiones:

1. Lo primero que hace la Corte en su proceso decisorio-resolutivo es determinar cuál es el resultado que considera justo y equitativo. Una vez determinada la solución equitativa, la Corte fabrica el raciocinio o el proceso que le lleve a tal solución.

2. La Corte le otorga a la equidad un valor decisorio de gran relevancia, al punto de que es la equidad el elemento determinante del resultado de las disputas, y no la herramienta que sirve para llegar a un resultado.

Las conclusiones anteriores nos llevan a entender que la Corte utiliza la equidad como el elemento esencial por excelencia en la preparación de sus decisiones, como lo haría un árbitro llamado a fallar ex aequo et bono, lo que no se condice con el rol auxiliar que esta debiese tener como elemento informante del derecho internacional.

\section{CONCLUSIONES}

Ningún autor puede menos que aplaudir el rol que cumple la CIJ en las relaciones entre Estados, no solo en su calidad de ente jurisdiccional si no que en particular en su forma de influir en la correcta conducta de los Estados y en la preservación de la paz y el derecho internacional. Lo anterior, no implica que todas las decisiones que tome la Corte sean perfectas ni que deban encontrarse exentas de críticas.

Los resultados de este juicio en particular pueden ser criticados de conformidad a la postura que asuma cada autor, pero se puede coincidir en que ciertos aspectos del fallo parecen arbitrarios y carecen de un real sustento en la evidencia presentada por las Partes o en el derecho internacional.

Somos de la opinión que la Corte erró al ponderar cada acuerdo entre las Partes por separado, puesto que la interpretación conjunta y sistemática de los acuerdos que reconocen la existencia de una jurisdicción inter partes hasta la milla 200 (Proclamaciones de 1947 y de Declaración de Santiago) con el Convenio de 1954 que reconoce la existencia de una frontera marítima entre ambos Estados, debiese haber conducido a la

\footnotetext{
38 International Court of Justice, Continental Platform Dispute (Alemania v. Holanda y Dinamarka), February 1969, Judgment; $\$ 92 “(.$.$) it is necessary to seek not one method of$ delimitation but one goal."
} 
conclusión de que Chile y Perú habían acordado una frontera marítima que corre por el paralelo geográfico que pasa por el Hito $\mathrm{N}^{\circ} 1$ hasta la milla 200 desde sus costas.

La elección del punto de término de la frontera acordada por las Partes parece más influenciada por la intención de la Corte de encontrar una solución equitativa, que no perjudique ni beneficie en exceso a ninguna de las Partes y que otorgue a cada una lo que la Corte considera que le corresponde por equidad, que por la evidencia y los argumentos presentados por los agentes y abogados de Chile y Perú. El raciocinio y fallo de la Corte son informados e influenciados a tal punto por la equidad y el fin al que quiere llegar la CIJ que pareciera que este fallo más que determinado por los elementos del derecho internacional fuera un fallo ex aequo et bono.

A pesar de que no pareciera correcto criticar a la Corte Internacional de Justicia por intentar ser justa y equitativa, no podemos dejar de estar de acuerdo con la posición del juez Orrego al señalar que, a pesar de que la equidad es generalmente aceptada como fuente del derecho bajo el Estatuto de la Corte, esta siempre ha considerado que el rol de la equidad se encuentra limitado por la ley como un tipo de equidad infra legem, esto es, bajo la ley y de acuerdo a ella, en oposición a una equidad preter legem o contra legem ${ }^{39}$.

En el fallo analizado, la diferencia entre fallar conforme a derecho y ex aequo et bono que hace el artículo 38 del Estatuto de la Corte parece oscurecerse y la línea que gobierna el actuar de la Corte en esta materia parece perderse. Es un camino muy pedregoso el que toma la Corte si es que los Estados que comparecen a ella esperando soluciones en derecho obtienen sentencias en equidad.

39 Orrego, Francisco; Partly Dissenting Opinion regarding the Maritime Dispute (Perú v. Chile), January 2014, Judgment; $\$ 23$. 